\title{
Urgences
}

\section{Les élégies d'Emmanuel Hocquard}

\section{Anne-Marie Clément}

Numéro 33, octobre 1991

Poésies parallèles : France - Québec

URI : https://id.erudit.org/iderudit/025673ar

DOI : https://doi.org/10.7202/025673ar

Aller au sommaire du numéro

Éditeur(s)

Urgences

ISSN

0226-9554 (imprimé)

1927-3924 (numérique)

Découvrir la revue

Citer cet article

Clément, A.-M. (1991). Les élégies d'Emmanuel Hocquard. Urgences, (33),

99-110. https://doi.org/10.7202/025673ar d'utilisation que vous pouvez consulter en ligne.

https://apropos.erudit.org/fr/usagers/politique-dutilisation/ 


\section{ÉTUDE}

\section{Les élégies d'Emmanuel Hocquard}

\section{Anne-Marie Clément}

Dans Les élégies ${ }^{1}$ d'Emmanuel Hocquard, le lecteur est confronté à un texte éclectique, énigmatique. D'abord, l'ensemble du recueil est haché par de multiples changements de sujet, de ton, de forme. C'est un édifice spatio-temporel démesuré qui est mis en place; des temps et des espaces étrangers s'entremêlent, portés par un mouvement de construction/ déconstruction qui constamment détruit et refait l'ordre des évenements: villes, édifices ruinés et rebâtis, tourbillon de personnages historiques ou anonymes. Le ton va du langage poétique à celui du compte rendu, guide de voyage, bulletin météorologique, et lexique des pancartes; il saute de la légende du conte («prestige et raideur d'une cotte de maille ${ }^{2}$ ) à la légende des cartes ( $\square$ maïs/betteraves/ $\square$ forêt de chênes/[...] $\square$ église romane* p. 34) La forme est tout aussi instable; le texte continu qui se lit ligne à ligne s'intercale au texte disloque, vertical; une typographie variable fait usage de l'italique, de lettres carrées, de parenthèses, de /, de \&, de lignes pointillées, de cases, de petits dessins, etc.; des langues étrangères s'inscrivent, parfois traduisibles, parfois intraduisibles.

À travers les sept élégies qui constituent le recueil, le récit d'une énigme insolvable se déplie. Les objets, les mots y prennent la valeur d'indices. Ils se dressent en liste; même la présentation des faits ressemble à une compilation. Les conditions de lecture sont déterminées par cette mise en scène; lecture de fouille, de dépistage qui scrute les frontières du temps, qui explore la nature de l'objet, du mot, et qui également questionne les frontières du texte, par une pratique peu connue aujourd'hui mais jadis populaire, la pratique du centon.

Dès le début du recueil Les élégies, le texte se joue contre le temps; la mise en place d'un infranchissable présent, d'un

\footnotetext{
1 Emmanuel Hocquard, Les élégies, Paris, P.O.L, 1990, 121 p.

2 Ibid., p. 34. Désormais, les références à ce livre seront indiquées dans le texte.
} 
100

temps "toujours terriblement intact ", la mise au silence du souvenir, visent la destruction de la structure même du temps. Le discours subira le même traitement; il sera disloqué.

Le retrait de la continuité du texte entraînera la destruction de son horizontalité et laissera place au vertige d'un texte lacunaire, vertical. Cette tendance à briser la linéarité du texte ira crescendo jusqu'à sa culmination dans "élégie 4 ". Lutilisation des espaces blancs, la dislocation du discours est telle que la lecture devient verticale, que les mots s'érigent: " tours d'esprit *, "silence d pic *, "pic de mots". Même la narration contribue à l'installatlon de cette verticalité: « à l'époque où $\mathrm{H} /$ fit construire la tour * (p. 57), «venant de recevoir / cette brique / qui l'assomma " (p. 58), «nous enfonçons / méthodiquement * (p. 58), « un dirigeable silencieux / surveillait / les sous-marins " (p. 65).

Cette élégie plonge dans le souvenir. Mais dans un temps contesté, le souvenir ne peut être que stérile; il y est surtout question de mort et d'exil. Alors même que s'installe la filature, "ainsi je vais réunissant les fils * (p. 62), elle est tout aussitôt, et par des assauts repétés, éconduite: d'abord, la filiation de la généalogie n'est présentée que pour etre brisée, les ancêtres meurent « et me laissent un orphelin* (p. 64); ailleurs, le fil s'use, "lorsqu'une corde fut trop usée / on relégua le hamac" (p. 66) ou tisse un piège, *et m'organisa comme un oiseau / dans un filet * (p. 66), jusqu'à ce que, à la fin, le fil lui-même soit perdu, *NOUS perdions ma trace / dans la pierre" (p. 68).

Dans ce recueil, le discours s'affiche comme discours hors du temps ( Mais le temps n'est pas la question $*$ p. 10); le passé, le présent sont ramenés en un même *espace *, ils se côtoient [« (spectacle de la main sur ces ossements si contemporains de la terre et de la main)», p. 27]. Mais le passé est toujours à l'état de ruines, d'ossements; il signifie ce qui est mort et toute tentative de le joindre à ce qui vit ne peut qu'échouer. Tout au plus, le travail de fouille ramènera au présent un passé ruiné, fragmenté, sans vie.

Cette vaste enquête menée auprès des objets, des hommes et des mots se poursuit jusque dans la dernière élégie; ultime tentative où le passé interpellé est un passé 
partagé: * RAPPELLE-TOI * (p. 97, 99), * te souviens-tu * (p. 106). La mélancolie de l'élégie se réfugie derrière cet acharnement à poursuivre l'inventaire. Mais inévitablement, la question se pose: dans cet excès de fragments, de traces, d'indices, où est la réponse? Que garder de cette accumulation de faits, de mots, de textes, de temps? «que faut-il taire? / l'énigme est: sans réponse * (p. 120). Accumuler les indices, examiner les traces n'aura pas servi à retrouver le fil. Le présent est et demeure infranchissable.

Dans l'ensemble du recueil, les objets matériels sont omniprésents. Des matières, des objets, des parcelles du paysage s'empilent dans les pages: terre, pierre, urne, fougère, dent, os, verre, aubépine, une coupe en verre rose, etc. Ils se déclinent en un seul mot ou alors en une «description minimale *, qui donne du relief au mot, accentue ses contours, le fait briller d'une présence re-marquée: "grues de fer jaune * (p. 10), « des cigarettes à la menthe * (p. 13), «le soleil de trois heures* (p. 99). Ces mots sont le plus souvent isolés sur une ligne, démontrant avec plus de force leur insularité.

Ce vaste inventaire prend appui sur des actions qui appellent l'énumération, la nomination: la fouille archéologique, le testament, et aussi l'enquête, ce * quelque chose à élucider pour de bon* (p. 11). Les faits comme les objets se présentent sous forme de liste, ils s'exposent plus qu'ils ne se racontent.

Dans ce contexte, même l'être humain semble participer au monde des objets. En effet, les choses et les hommes, une fois rassemblés, finissent par se ressembler: «enfants au visage bien lavé / dalles brillantes * (p. 31). Ainsi en est-il de ces * hommes de la pénombre * avec * une dent en or/dans leur bouche», aperçus dans un café de pénombre et d'éclat, se moulant au décor: "le marbre gris des tables et les miroirs lavés au blanc d'Espagne* (p. 17). Il est dit aussi que le *bruit de leurs voix * s'accorde au *bruit de leurs verres *. Le marbre, l'or, le verre, les miroirs; les hommes se figent et le temps se fige aussi puisque «jamais aucun d'eux ne mourait * (p. 17)

Mais alors que le temps s'immobilise, le mot se secoue, se déplace; il devient terrain d'exploration, mot-objet, mot-texte: 
102

*br isait* (p. 63)

* sombr

e $\quad s *($ p. 58)

* dans son erm

i

tage de roses $*($ p. 57)

Dire «brisait * en brisant le mot, faire sombrer le mot * sombre *, bâtir l'ermitage à partir du mot, ces procédés disent la présence matérielle, graphique des mots, le *compte-tenu des mots ^. Par ces procédés mimologiques, le mot cherche à signifier par delà son signifié. Ainsi, dans ce texte (p. 28),

peuc peuc peuc peuc

le moteur à un cylindre d'une barque dans le détroit

l'onomatopée, la répétition du mot ainsi que son graphisme, donnent une image multi-dimensionnelle de la barque. Représentations sonore, mobile, et visuelle (jusqu'au jambage du $p$ semblable à l'hélice du moteur dans l'eau) convergent. Les trois référents de la ligne suivante se concrétisent: le moteur à un cylindre par le bruit, la barque par sa forme dessinée et le détroit par le parcours de la barque qui se déplace. L'effet de mouvement est amplifié par le passage de la barque sur la page suivante: "Ce soir les barques reprendront la mer" (p. 29). Une fois cette composition mise en place, l'image du premier vers s'impose si bien que le second vers s'apparente à une traduction ou encore à un titre de tableau.

Quelques mots suffisent pour remettre en question le sens du discours. Ainsi en est-il de ce texte (élégie 2, I, p. 2325) qui raconte un été de fouilles archéologiques. Les os et les tombes font partie de l'histoire, ils prennent place parmi les urnes, les vases, les monnaies. Mais en même temps, ils «font signe $»$. Les *os, / os, os, os, os » lancent des SOS alors que le danger, celui d'une chute, se lit dans « les tombes, les tombes, les tombes de la falaise*. À partir de la répétition des os et des tombes, une autre version se construit, une sub-version qui laisse poindre un danger. Sous cet éclairage nouveau, le saccage et la profanation du travail de la fouille sont mis en relief: "crâne brisé *, " tombe à moitié ouverte ", à quoi il faut ajouter cette remarque mise entre parenthèses: "Cela méritait-il le travail de tout un été? " La linéarité du texte, 
son horizontalité ponctuée de lignes pointillées, la page comparable à une vitrine où tout est aligné sur des tablettes, * derrière des étiquettes probablement précises * est donc ébranlée par les insinuations des os et des tombes qui instaureront la verticalité du cri et de la chute.

Le double sens des mots peut également permettre le redoublement du discours par un autre discours. Dans "élégie 4 », ce texte écrit en italique, se lit comme texte et se relit comme métatexte:

ce sont les plaques romaines

trouve sa pleine valeur depuis

dont la matiere

qu'elles ne nous servent plus

a savoir

combien de genéraux moururent

dans le combat

ou quel chemin nous mène

au forum ou au marche (p. 61)

Isolé par son caractère typographique différent, il se présente comme une plaque textuelle, et comme une plaque romaine, puisque «italique » signifie aussi: de l'Italie ancienne. On y lit que le message écrit masquait la pleine valeur de la matière première de la plaque. Dans un recueil qui met en évidence la pleine valeur du signifiant des mots, un tel texte peut également se lire comme un commentaire sur la matérialité des mots. D'ailleurs, ce double usage du texte est en quelque sorte supporté par le double sens du seul mot matière qui signifie à la fois le matériau et le sujet d'un ouvrage, et qui laisse comprendre que derrière la matière, se trouve la matière. De fait, un tel dédoublement du mot se rencontre fréquemment dans le livre, laissant le lecteur dans l'incapacité de distinguer s'il s'agit du même ou de la différence, d'une coïncidence ou d'une discordance: «des fleurs / furent délivrées de la végétation qui les masquait» (p. 82); "en train de reconstruire / du rêve que / tu avais rêvé la nuit d'avant * (p. 68); * voir/comme le nom / [...]/ avec dessus ÉCRIT encore lisible le nom * (p. 52); * (comme le moment précis où la rivière est vraiment/ rivière)* (p.9).

Les références à l'Histoire, particulièrement l'histoire de l'Empire romain dans laquelle baigne entièrement * élégie 6 », 
mais également à l'histoire littéraire, sont très nombreuses et très variées; présentes comme un assemblage de noms, de faits, de dates auxquels se mêlent œuvres d'art, peintures, statues, architectures. Face à cette mémoire défaillante de l'Histoire, la lecture prend parfois des allures de fouille ou d'enquête.

Provins occupe une place particulière dans le recueil; pour autant que l'on connaisse Provins (connaissances du type *guide touristique *) une foule d'indices eparpillés dans le texte ramènent sans cesse à cette ville: ville d'origine galloromaine (Pruvinum); ville "verticale " qui se divise en une ville haute entourée de remparts et une ville basse; ville très riche en monuments, Tour aux Engins, Tour César, ancien palais des comtes de Champagne, musée archéologique (Grange-aux-Dîmes), relique (Saint-Ayoul); aussi, le lycée dans l'ancien palais, les machines agricoles, les roseraies, les carrières souterraines de pierres réfractaires, les glaisières fournissant les matières premières aux potiers, tuiliers, briquetiers; Nangis et Longueville, deux villes voisines, etc. La configuration de Provins, sa géographie, son histoire ancienne et actuelle, reprennent la configuration du recueil, les faits relatés comme les thèmes développés: la falaise, la fouille archéologique, les ruines romaines. Le portrait de Provins constitue une condensation, une sorte de mise en abyme de la grande fresque peinte dans le recueil.

Toute lecture est bel et bien le lieu d'un travail de décryptage et cette assertion prend une ampleur particulière dans *élégie 6 *. Celle-ci a trait surtout à l'histoire de l'Empire romain, histoire humaine et histoire de l'art confondues. Les renseignements sont à la fois très précis et non identifiés, ce sont des faits qui supportent à la fois le poids de l'évidence et l'incertain de l'anonymat. Voilà qui constitue en soi une énigme et invite à un travail de fouille. Mais ce travail est tout aussitôt découragé par la mise en scène invraisemblable qui mine toute la confiance qui pourrait se bâtir à partir de ces détails qui paraissent si "vrais". Voici les premiers vers de cette élégie:

À l'époque

où

il fit commencer les travaux, l'île était accessible 
On voit encore le môle

par de petits ponts mobiles

bordés de docks et d'entrepôts

où se dressait le phare (p. 81)

Rapidement l'évidence de l'impossibilité de la reconstitution s'impose; il ne sera plus jamais question de l'île, des ponts, du môle ou du phare (remplacés par le jardin, la statue d'une fillette, les deux bancs latéraux, le grand escalier, etc.), alors que la récurrence de l'époque, des travaux ou du "il », ne fera qu'alimenter l'ambiguïté de leur signification. Le «il $»$ qui habite toute l'élégie est non seulement anonyme, c'est un *il n impossible: tantốt une personne, tantôt impersonnel, tantôt un objet, tantôt une autre personne. Et entre les faits relatés, les objets présentés, les liens ne sont pas inscrits. L'élégie se présente donc comme un assemblage de vers sans suite.

Cette élégie est effectivement un collage de fragments épars. J'ai identifié une soixantaine de citations, phrases ou bouts de phrases du Guide vert Michelin sur Rome (voir la liste à la fin). Le nombre d'extraits est tel que l'hypothèse d'un texte entièrement constitué à partir d'un collage de fragments de ce guide est plausible; une "fouille " plus complète permettrait de vérifier cette hypothèse. Ce procédé rappelle la pratique du centon qui fut populaire durant l'Antiquité, greffant ainsi à ces élégies, version moderne, une version tout aussi moderne du centon.

Cela fait d'^élégie 6 » un texte construit formellement à même les ruines d'un autre texte, de même qu'un texte construit thématiguement à même les ruines d'une ville, à même un temps ruiné. Un tel usage de la citation suscite des questions; pourquoi cette fiction du Michelin? La fin de l'élégie précédente préparait ce discours: «User des mêmes mots sera notre manière / de nous taire sans avoir l'air de laisser mourir / la conversation » (p. 76).

Maintenant, nous sommes en mesure de découvrir que le «i l» est à la fois Néron, Claude, César, statue d'Aristote, Chateaubriand, Caligula, un proverbe, une ville. Nous sommes en mesure de reconnaître les ruines de tel édifice, les traits de telle statue. Chaque citation possède une signification, chacune possède sa vérité. Cependant, l'assemblage de ces citations fait surgir d'autres sens. Il faut souligner que les 
liaisons entre les diverses citations ne s'écrivent pas; ces liens se mettent en place par simple effet de contiguité spatiale. Dans ce texte sans ponctuation et dont le découpage métrique est irrégulier, imprévisible, la lecture crée des liaisons;

sur le vernis noir des vases

blancs

jaunes

rouge sombre

des fleurs (p. 82)

furent délivrées de la végétation qui les masquait (p. 82)

Ainsi, par-delà le discours sur les poteries de la villa Gulia (du début de la citation jusqu'à "fleurs *) continué par celui sur les fouilles entreprises à la villa d'Hadrien (dernier vers de la citation), le sens est dévié vers cette végétation (les fleurs) délivrée de la végétation qui la masquait. C'est donc par la mise en scène du texte que les liaisons se forment. Cette écriture délivre le discours de ses lieux communs, délie la langue des mots, s'ouvre à d'autres propositions de signification.

La mémoire défaillante de l'Histoire et celle de l'histoire individuelle (le souvenir) redisent l'impossible retour du passé dans ce présent « infranchissable . Les liens sont coupés. La déconstruction du récit, de la phrase, du mot, résulte de cette remise en question des liaisons. En fait, la construction de cette élégie s'apparente tout à fait au * trompel'œil ^ (p. 90 et 93), à « l'architecture à rebours * (p. 90) dont il est question dans le texte. Ces termes rappellent la configuration essentiellement spatiale de ce recueil dans ce qu'il dit (qui va jusqu'à la spatialisation du temps) et dans sa façon de le dire (l'organisation de la page).

Les elégies se présente comme un texte fragmenté, mais aussi comme un recueil construit: collage de faits et d'objets hétéroclites, collage de citations; ce qui convoque une lecture où la seule certitude loge dans ces objets que l'on peut nommer, compiler. Et pourtant, à travers toutes ces différences, il s'y construit une certaine homogénéité. Car c'est un recueil qui, malgré l'achronie avouée, raconte une intrigue, suit les traces et cherche à résoudre le drame. L'énigme, la fouille, actionneront cette quête d'indices dans l'expression (lié-délié des phrases, des mots) et dans le contenu (lié-délié des faits 
et personnages) du texte. Et si le dénouement tient de la plainte, c'est que l'entreprise était condamnée d'avance. Le livre se fermera tendrement sur ces mots : «avait également trait / à la vie / toi / \& ton regard/: / beaux / oui si beaux / yeux grand / OUVERTS * (p. 121). Il faut se demander si le présent et le passé ne cherchent pas une dernière fois leur point de rencontre dans cette ultime vision des yeux grand ouverts, vision qui oscille entre la vie et la mort.

Fragments du Guide de Tourisme Michelin, Rome, 4* édition, 1984. repris dans * élégie 6 * (p. 81-84). Les extraits sont en caractère gras. Ils sont présentés selon leur ordre d'apparition dans le recueil.

Théâtre maritime, villa d'Hadrien. L'île était accessible par de petits ponts mobiles. (p. 201)

On voit encore, au Nord-Ouest du musée, le môle où se dressait le phare. (p. 209)

Dans une vitrine, est exposé un crabe en porphyre vert, pierre rare. (p. 82)

\section{un fragment de la Sainte Lance (p. 77)}

Salle de la Cheminée. Cette salle abrite une belle collection d'antéfixes. (p. 58)

La Danaé de Corrège qui illustre le mythe de Zeus pénétrant chez Danaé sous la forme d'un nuage. (p. 176)

Pasquin, la célèbre "statue parlante" (p. 98) [Au MoyenÂge, groupe de statues qui recueillaient les inscriptions satiriques dirigées contre les puissants de la ville. Statue de Madame Lucrezia, Pasquin, Marforio et l'Abate Luigi.]

la Grâce du Palatin [...]: son vêtement, très fin, semblable à une " draperie mouillée ". (p. 119)

sur le vernis noir des vases, l'artiste peint en blanc, en jaune, en rouge sombre des fleurs (p. 171)

[Villa d'Hadrien, fouilles 1870]. Peu à peu des voûtes [...] apparurent, délivrées de la végétation qui les masquait. (p. 201)

Au Moyen-Âge, il [Marforio] faisait partie du groupe des "statues parlantes" (p. 60) 
108

Les archéologues ont interprété cette scène comme la naissance d'Aphrodite, déesse de l'Amour née de la mer, entourée des Heures dont les pieds effleurent les galets de la plage. (p. 119)

[Chateaubriand, ambassadeur de la France, à Rome]. «mais je ne m'étais pas douté de ce que pouvaient être des fêtes à Rome: elles ont quelque chose de la poésie antique qui place la mort à côté des plaisirs » écrivit-il. (p. 100)

Tivoli. Des carrières, des centrales hydro-électriques, des papeteries, des industries chimiques sont ses activités principales. (p. 201)

[Peinture de Raphaël] Aristote, la main tournée vers le sol, signifie que l'idée n'a d'existence que dans la chose matérielle (p. 85)

Il [Caligula] se plut à faire niveler les montagnes et élever les plaines, et souhaita un massacre, une famine, un cataclysme qui ferait passer son règne à la postérité; (p. 17)

La Chasse de Diane illustre bien le savoir bolonais (remarquer la précision des arbres, l'oiseau atteint par les flèches) (p. 175)

Dans la nuit du 15 au 16 juillet 1823 [incendie de la basilique Saint-Paul-Hors-les-Murs] (p. 141)

flotteurs de filets de pêches, cordages, clous (p. 209)

La chapelle des reliques abrite [...] deux épines de la Couronne; [...] un clou de la Passion. (p. 197)

L'Aventin fut entièrement dévasté. (p. 192)

un lieu sacré [la Curie] à l'intérieur duquel un augure pouvait communiquer aux hommes la volonté des dieux qui lui était transmise par certains signes: le vol des oiseaux, l'appétit des poulets sacrés, les petits faits inhabituels. (p. 41)

Claude, après un transport au cerveau, ne comprit plus les choses que très lentement; "en voyant ses funérailles, il comprit qu'il était mort " dit Sénèque. Néron, monstrueux, se prit pour un homme de théâtre; "quel artiste va périr avec moi!" dit-il avant de se donner la mort. (p. 17) 
[Cicéron] il meurt égorgé par les tueurs à gages des triumvirs. (p. 42)

lorsque la nouvelle de son assassinat se répandit [Caligula], personne n'osa se réjouir, craignant un piège. (p. 17)

le proverbe signifiant qu'il y a peu du triomphe à la chute ignominieuse (p. 56)

À cette époque [1870] disparut le port de Ripetta (p. 163)

P. Cornelius Scipio s'embarqua pour l'Espagne (p. 205)

[Ostie] les quartiers résidentiels s'étendirent vers la mer. (p. 205)

Église Sainte-Marie-de-la-Conception. [...] dalle funéraire [...] portant l'inscription * Hic jacet pulvis, cinis et nihil " (Ici gît de la poussière, des cendres, rien (p. 177)

un char miniature (p. 170)

* le neuvième jour avant les calendes d'octobre * [naissance d'Octave] (p. 47)

[Néron] contempla l'incendie qu'il avait lui-même ordonné d'allumer, "charmé par la beauté des flammes ", d'après le récit de Suétone. (p. 131)

[Constance II, devant le forum de Trajan, selon l'historien Marcellin]: «il resta stupéfait » (p. 130)

Saül entendant la voix du Christ: «Saül, Saül, pourquoi me persécutes-tu? ? (p. 140)

Théoricien de la perspective, A. Pozza a mis son talent au service du trompe-l'oil (p. 106)

Les critiques parlèrent d'architecture «à rebours». (p. 107)

[Octave] il agrandit sa maison puis la reconstruisit après qu'un incendie l'eut détruite (p. 97)

[maison de Néron] une pièce d'eau « semblable à une mer" (p. 67)

Circus maximus. (il y avait quatre écuries distinguées par une couleur) (p. 193)

Pasquin fut la "statue parlante" la plus bavarde de Rome. (p. 159) 
le portrait de Béatrice Cenci par Guido Reni (1575-1642) est remarquable de délicatesse; Stendhal, [...] dans ses Chroniques italiennes, a écrit de ce portrait * la tête est douce et belle, le regard très doux.... (p. 189)

Paisiblement étendu, le colosse semble s'ennuyer un peu à contempler l'eau de cette vasque. [Marforio, statue parlante] (p. 60)

"Domine quo vadis? (Seigneur, où vas-tu?) lui demandat-il [Saint-Pierre]. (p. 143)

On blâma César parce qu'il lisait son courrier à l'amphithéâtre. (p. 68)

Pasquin (une autre statue parlante). (p. 60)

[César] II lance une flotte à leur poursuite et les anéantit. (p. 16)

Rome remportait à Antium sa première victoire navale. (p. 205)

Aujourd'hui le Palatin est extrêmement ruiné. (p. 47)

En 1673, il [Bernin] exécutait la maquette en terre cuite pour la statue équestre de Louis XIV. (p. 176)

Plusieurs archéologues se sont accordés pour attribuer ces temples à Junon, à Janus et à l'Espérance. (p. 150)

Léon X s'éteignait saisi d'une " petite fièvre ". (p. 190)

[colonne de Marc-Aurèle] raconte les épisodes marquants de ses guerres en une série de bas-reliefs enroulés en spirale. (p. 163)

De Caravage, admirer aussi le douloureux David montrant la tête de Goliath. (p. 176)

Ce bateau fut volontairement coulé. (p. 209)

Le gouffre se referme et un petit lac subsista. [légende du soldat qui s'était jeté dans le gouffre après verdict d'un augure]. (p. 43)

Hercule, le héros paré d'une peau de lion.(p. 68)

Diogène le Cynique, plein de mépris, est à demi étendu sur l'escalier.(p. 85)

Les deux cavaliers sont représentés debout, à côté de leur chevaux (p. 56). 